\title{
Development of a Simple Probe for Non-Destructive Reversible Electric Contact to nm-Thick Films and 2D Films
}

\author{
Michiko Yoshitake* and Shinjiro Yagyu \\ Nano-Electronics Devise Materials Unit, National Institute for Materials Science, Sakura 3-13, Tsukuba 305-0003, Japan \\ Toyohiro Chikyow \\ Nano-Electronics Devise Materials Unit, National Institute for Materials Science, Namiki 1-1, Tsukuba 305-0044, Japan
}

(Received 1 April 2015; Accepted 10 May 2015; Published 20 June 2015)

\begin{abstract}
A simple probe that is applicable as an electric contact to nm-thick films and 2D films such as graphene and $\mathrm{MoS}_{2}$ without destroying the specimen has been developed. The concept of the development of the probe is based on the repulsive region used in atomic force microscopy technique but without any precise feedback. The robust electric contact with the probe has been demonstrated by biased XPS measurement of a MOS specimen and by resistivity measurement of a 5-layer graphene film on sapphire. It has also proved that there was no detectable damage or contaminations on the specimens after the measurements.
\end{abstract}

[DOI: 10.1380/ejssnt.2015.307]

Keywords: Electrical transport; Schottky barrier; MOS structures; Photoelectron spectroscopy; Polycrystalline thin films

\section{INTRODUCTION}

Recent downsizing of metal-oxide-semiconductor (MOS) transistors requires the replacement of current materials in use. In order to overcome the limit with silicon dioxide, insulators called high-k materials are extensively explored [1-3]. The replacement of insulators is causing problems at interfaces both between high- $\mathrm{k}$ and gate electrode [4,5], and high-k and $\mathrm{Si}[6,7]$. In contrast to a poly-Si/SiO $/ \mathrm{S}_{2} / \mathrm{Si}$ substrate MOS structure, the electrical properties of a high-k MOS cannot be controlled according to the ideal Schottky-Mott relationship. To understand the cause of the deviation from the ideal relationship, separate measurement of electronic potential at two interfaces, gate metal/high-k insulator and high-k insulator/Si, is desired, which is difficult with electrical measurements. We have developed XPS technique under bias voltage between a gate electrode and a silicon substrate for the separate characterization of two interfaces $[8,9]$. In order to have signals from high-k insulator locating below the gate, the gate film has to be nm-thick, which is too thin to get an robust electric contact without damaging the gate metal. Therefore, in the previous measurement, a sample with a special shape (Fig. 1) was fabricated for the measurement. To avoid the need to fabricate samples with a special shape like in Fig. 1, XPS measurement with hard X-ray using synchrotron radiation has been developed, which has a large escape depth of photo electrons. However, a large escape depth means less depth resolution.

Therefore, we developed an electric contact on nmthick film without damage so that interface analysis is possible with conventional XPS without fabricating a specially shaped specimen like in Fig. 1. Furthermore, non-destructive electric contact to nm-thick films enables other electronic devices with two-dimensional materials such as graphene and $\mathrm{MoS}_{2}$, which attract great attention for gate materials with high mobility $[10,11]$.

\footnotetext{
* Corresponding author: yoshitake.michiko@nims.go.jp
}

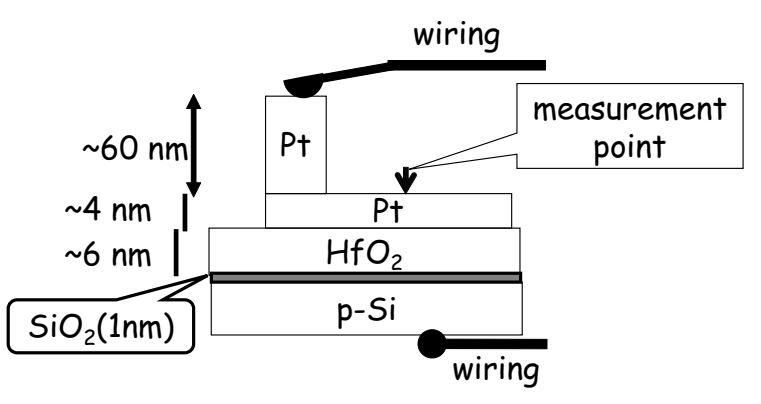

FIG. 1. Structure of the specimen used for bias-XPS in the previous measurement.

\section{CONCEPT OF NON-DESTRUCTIVE ELECTRIC CONTACT}

At the beginning, the principle of electric contact without destroying a nm-thick film is considered from a physical point of view. A condition that can be regarded at electric contact is examined by assuming contacting materials to be a metal. Based on electronic theory, electrons in the two metals in contact should be at equilibrium (Fermi level of the two metals aligns). This situation is realized by the overlap of electron orbitals of the two metals. The degree of the electron overlap can be monitored by a force curve in atomic force microscopy (AFM). A typical force curve is shown in Fig. 2. In the repulsive region, electrons of the tip and the sample overlap and I-V characteristics is measured in this region. Because a force curve at repulsive region usually shows repeatable trace when the cantilever approaches and retracts, which means the elastic deformation in the repulsive region and complete recovery of the sample and the tip, AFM is regarded a non-destructive method. Therefore, non-destructive electric contact is realized when two metals are in contact under repulsive region but still in elastic deformation range. A typical force applied in the repulsive region is calculated using a force curve on $\mathrm{Cu}$ measured with a cantilever with a force constant of 3 $[\mathrm{N} / \mathrm{m}]$. Elastic deformation was observed at approximately $5 \mathrm{~nm}$ displacement. The radius of the contact 


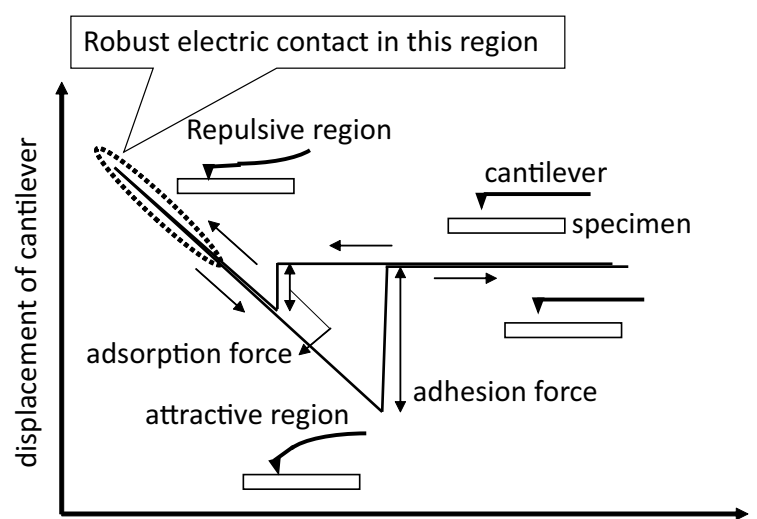

Tip-specimen distance

FIG. 2. Condition for electric contact in AFM force curve.

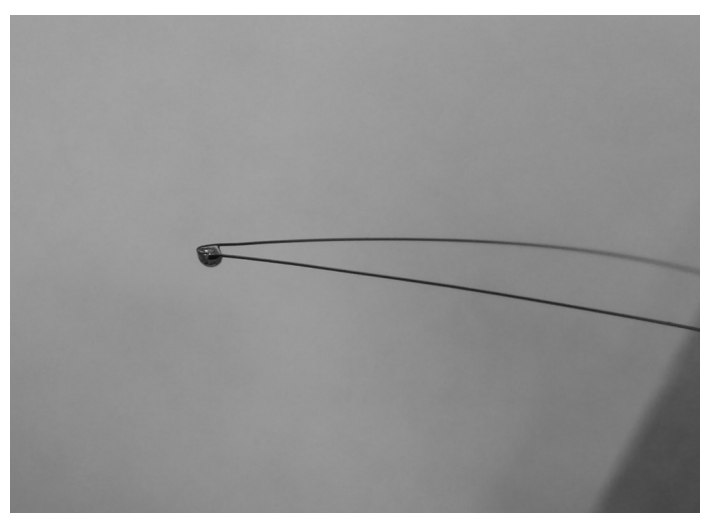

FIG. 3. Photograph of a developed contact probe.

area of the cantilever is estimated to be $10 \mathrm{~nm}$ from the lateral resolution of AFM images. Therefore, the pressure is $\{3[\mathrm{~N} / \mathrm{m}] \times 5[\mathrm{~nm}]\} /\left\{\pi \times 10\left[\mathrm{~nm}^{2}\right]\right\} \sim 5 \times 10^{7}[\mathrm{~Pa}]$.

Using AFM apparatus, however, is not practical for the measurement of electrical property of most of devices in the following reasons: The contact area in AFM is nearly atomic and to keep the force in the proper range, precise real time control of both lateral and vertical positions and anti-vibration device are essential.

A probe that can be used for non-destructive electric contact should have larger contact area so that the deviation of force would be diluted. In order to realize the corresponding pressure to AFM with a larger contact probe (a radius of the contact area of $100 \mu \mathrm{m}$ ), the force to be applied is $5 \times 10^{7}[\mathrm{~Pa}] \times\left\{\pi \times(100 \mu \mathrm{m})^{2}\right\} \sim 1.5[\mathrm{~N}]$. Using a commercially available spring plate with a thickness of $0.5 \mathrm{~mm}$, the displacement with a force of $1.5[\mathrm{~N}]$ is approximately $1 \mathrm{~mm}$, which can be easily detected by eyes.

\section{FABRICATION OF A PROBE FOR NON-DESTRUCTIVE ELECTRIC CONTACT}

To meet the pressure requirement for a non-destructive electric contact, a probe with an appropriate contact area and spring constant should be developed. Once an appropriate contact area is decided from measurement point of view such as the size of the specimen, the size of the con-

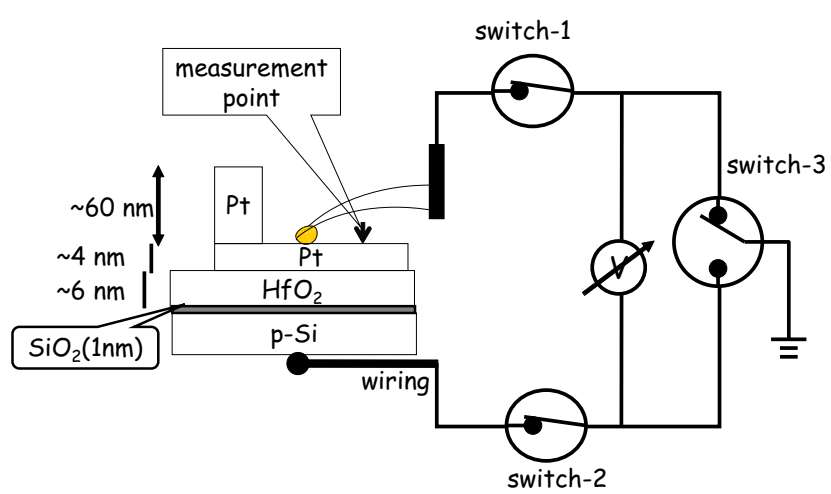

FIG. 4. Experimental setup for bias-XPS measurement with the developed contact probe.

tacting ball and the spring constant of the support of the ball are fixed. Details are found in the ref. [12]. In Fig. 3, a photograph of a probe is shown as an example. In this case, a $70 \mathrm{~mm}$-long tungsten wire with a diameter of 0.10 $\mathrm{mm}$ was bent at the center to make a hairpin shape, a 10.2 mm-long gold with a diameter of $0.15 \mathrm{~mm}$ was tied at the bent of the tungsten wire. Then, the gold-tied tungsten wire was resistively heated in vacuum to melt the gold, resulting in a gold ball. We chose to make a contact ball by melting because smooth surface is preferable not to damage a specimen upon electric contact. The size of a gold ball can be controlled by the amount of gold wire set on the tungsten wire before melting it in vacuum. The radius of the contact area was estimated about 100-150 $\mu \mathrm{m}$ from optical microscopic observation for the ball fabricated in the above condition. The material for a contact ball is not limited to gold: we fabricated copper and aluminum balls in a similar way, where the melting point of ball materials is rather low. In order to make a contact ball with high melting temperature materials or volatile materials, depositing those materials on the gold ball works. We applied the deposition technique to fabricate a Pt ball. The spring constant of the support can be controlled by the thickness, length and shape of the support.

\section{BIAS-XPS USING THE NON-DESTRUCTIVE ELECTRIC CONTACT}

In order to test the non-destructive electric contact described above, a Si-MOS structure used for the previous bias-XPS experiment [9] is used. This time, instead of having a contact to $\mathrm{Pt}$ thick film, a gold ball supported by a tungsten wire is pushed toward a $4 \mathrm{~nm}$-thick $\mathrm{Pt}$ film. The arrangement of the sample, contact probe and the electric circuit is shown in Fig. 4. The measurement procedure of XPS spectra is exactly the same as before. In Fig. 5(a), XPS spectra under various bias voltages are shown, which are compared with the previous results (Fig. 5(b)). When Pt is grounded in Fig. 4, the binding energy of $\mathrm{Pt} 4 \mathrm{f}$ should stay the same regardless of bias voltage if the contact ball has a perfect electric contact with 4 nm-thick Pt film, which is proved in Fig. 5(a)-(i). The shifts in Hf $4 f$ and $\mathrm{O} 1 s$ according to the bias voltage are due to the property of the specimen as observed 
a) Contact with developed probe on 4 nm-thick Pt film

i) Pt-grounded
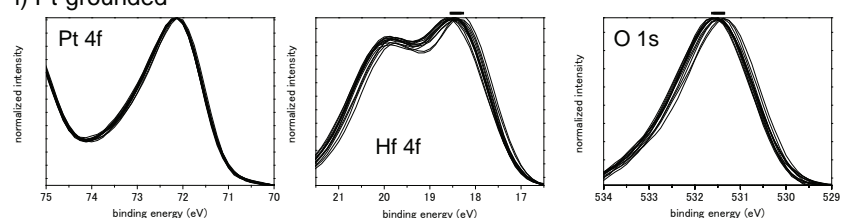

ii) Si-grounded
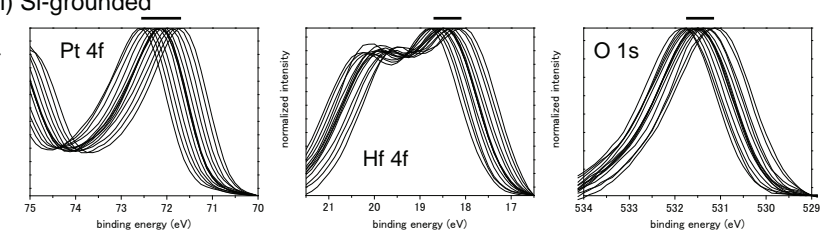

b) Contact on $60 \mathrm{~nm}$-thick Pt film (previous results)
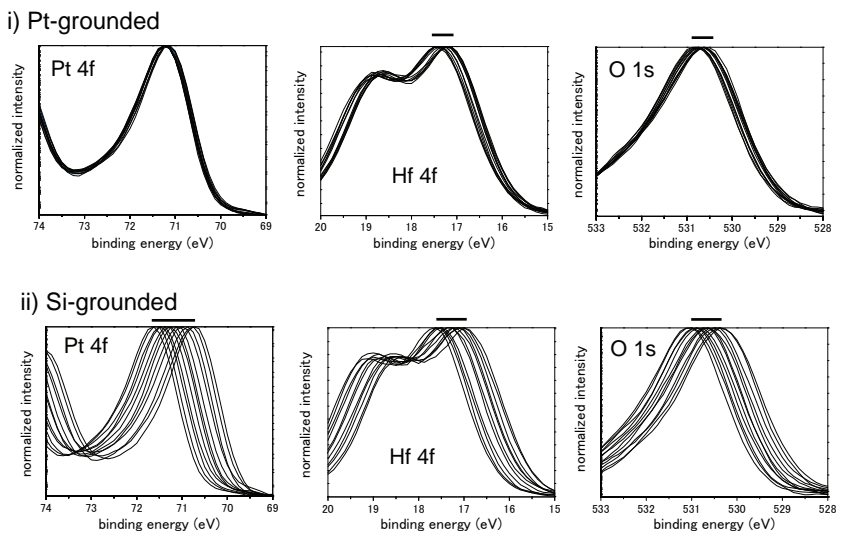

FIG. 5. Changes in XPS spectra by applied bias voltages: a) using the developed probe, b) previous results with contact on thicker electrode.

in the previous experiments in Fig. 5(b)-(i). When Si is grounded and bias voltage is applied to Pt film in Fig. 4, the binding energy of $\mathrm{Pt} 4 f$ is expected to shift according to the applied bias voltage if the contact ball has a perfect electric contact with $4 \mathrm{~nm}$-thick $\mathrm{Pt}$ film. This is again proved in Fig. 5(a)-(ii). The shifts in Hf $4 f$ and $\mathrm{O} 1 s$ according to the bias voltage are less than $\mathrm{Pt} 4 f$, which again is attributed to the property of the specimen as observed in the previous experiments in Fig. 5(b)-(ii). The peak shifts in $\mathrm{Pt} 4 f$, Hf $4 f$ and $\mathrm{O} 1 s$ as a function of bias voltage are plotted in Fig. 6(a) and are compared with the corresponding previous results (Fig. 6(b)). The scale in Fig. 6(a) and (b) is the same. Data points in Fig. 6(a) is not the same as in Fig. 6(b), but the slopes and deviations of the data are almost the same. Therefore, we can conclude that the proper electric contact is realized with the gold ball. This also means that the 4 nm-thick Pt film was not destroyed by the contact. If the thin Pt film underwent destruction and have cracks, the position of the XPS measurement would not be at the potential of applied voltage. In these experiments, electric contact was made only to apply bias voltage and no electric current flew in the circuit, resulting in no influence of a)
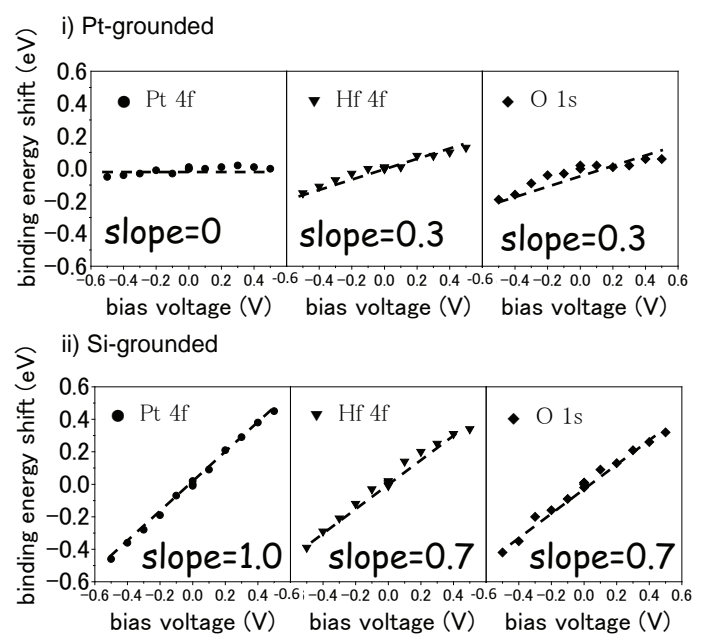

b)
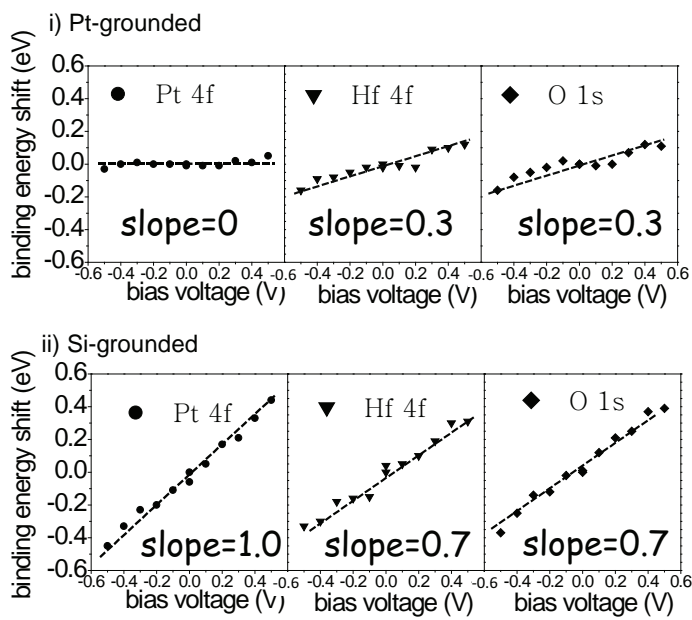

FIG. 6. Binding energy shifts in XPS spectra by applied bias voltages: a) using the developed probe, b) previous results with contact on thicker electrode.

contact resistance between $\mathrm{Pt}$ film and the contact both in the previous experiment (Fig. 5(b) and Fig. 6(b)) and in this experiment with the gold ball contact (Fig. 5(a) and Fig. 6(a)).

We also checked that there is negligible contamination from a probe by electric contact. Figure 7(a) shows the photo of the sample with the indications of the contact site of the Au ball where the contamination was checked by XPS. The site indicated as XPS measurement in the figure is for the measurement shown in Fig. 5(a). Au wires at the left side of the contact position are touching to the $60 \mathrm{~nm}$ thick $\mathrm{Pt}$ film to make the potential of the $\mathrm{Pt}$ film grounded through the In plate on the top for XPS measurement. The solid line in Fig. 7(b) is the measured XPS spectra, where the position of $\mathrm{Au} 4 f$ doublet is shown in dotted line in the inset. As displayed in the figure, no detectable peak from Au was observed. This suggests that making electric contact causes no influence on the specimen so that the specimen can be used for further experiments, which is quite different from commonly adopted electrodes made by metal deposition on nm-thick films. In a sense that the specimen returns to the condition before electric measurements, we call this as "reversible" contact. 


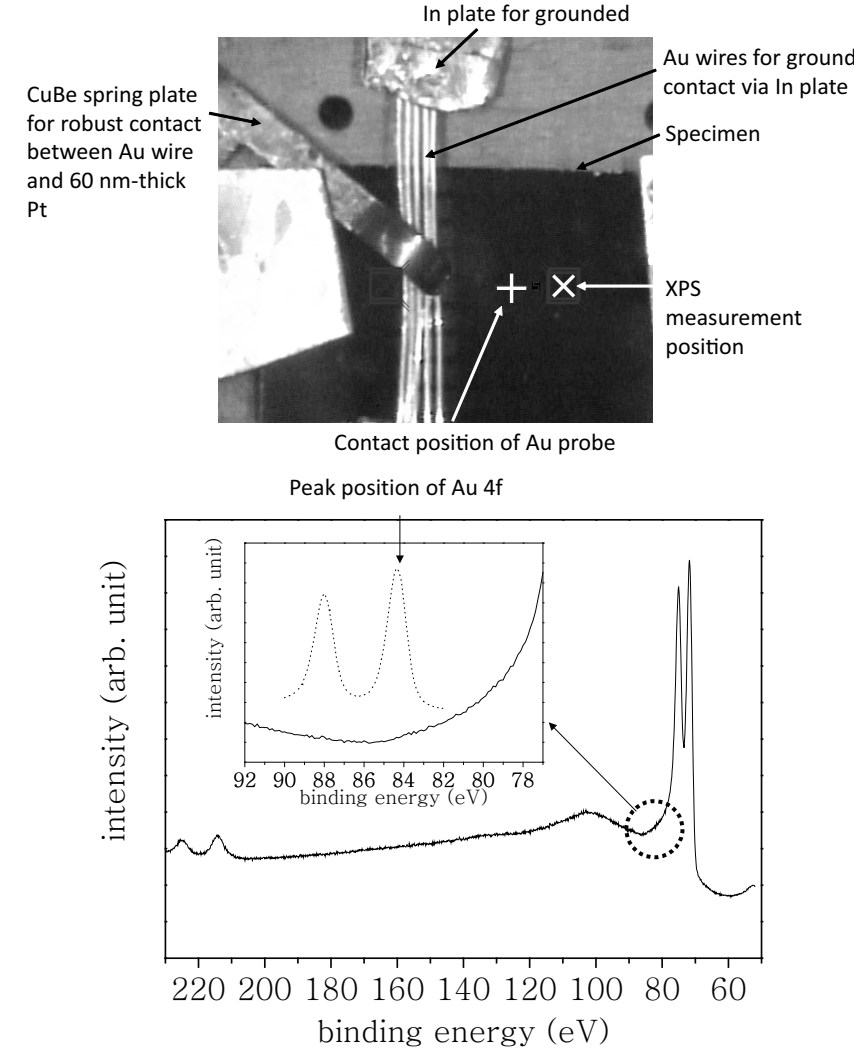

FIG. 7. Photograph of the specimen for checking contamination by the contact (a) and XPS spectra of the contact position after contact (b). a)

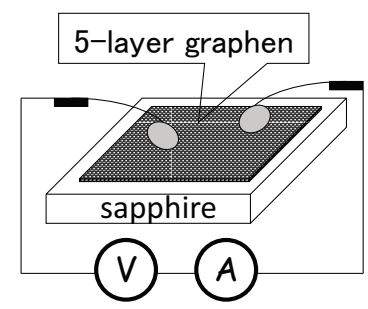

b)

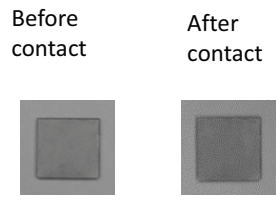

FIG. 8. Schematic illustration of resistance measurement on graphene using the developed probes (a), photographs of the graphene before and after the resistance measurement (b).

\section{RESISTIVITY MEASUREMENT OF 5-LAYER GRAPHEN}

The validity of the Au ball for resistivity measurement of fragile ultra-thin layer has been tested using 5-layer graphene transferred to sapphire [13]. Two Au balls were pushed toward the graphene closely with a contact distance of approximately $1 \mathrm{~mm}$ apart. Figure 8(a) schematically illustrates the measurement arrangement. The resistivity measured by a conventional two probe method was $3 \mathrm{k} \Omega$, giving the resistivity of approximate $8 \times 10^{-6}[\Omega \mathrm{m}]$. This value appears reasonable because the resistivity of graphite (= infinitely thick graphen) is reported as 3-

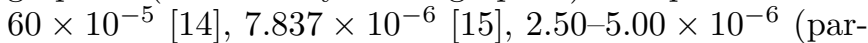
allel to the basal plane) $[16], 4.0 \times 10^{-7}[\Omega \mathrm{m}][17]$, that of 2-10 layer-reduced graphene oxide is $8.8 \times 10^{-5}[\Omega \mathrm{m}]$ [17], and the possible lowest resistivity of graphene estimated from the atomic vibration is $1.0 \times 10^{-8}[\Omega \mathrm{m}][18]$. The absolute value of the measured resistivity is not accurate because the influence of contact resistance between $\mathrm{Pt}$ film and a probe is not cancelled in the measurement with the conventional two probe method. However, the above measurement is considered enough to show that the developed probe is applicable for resistive measurement of 5-layered graphene and four probe method with four gold ball probes should work for accurate resistance measurements.

In Fig. 8(b), photos of the graphene specimen on sapphire before and after the measurement are shown. There is \pm 1 layer thickness distribution in the purchased specimen and the deviation was visible as a grey scale pattern by eyes before the resistance measurement. After the measurement, the same grey scale pattern by the thickness deviation was observed by eyes, indicating no detectable damage by the electric contact with the probe even in one graphene layer level. This demonstrates that resistivity measurement with the probe is possible without damaging 5-layer graphene.

\section{CONCLUSIONS}

A new type of non-destructive electric contact probe has been developed by realizing a repulsive force condition with atomic force microscopy in microscopic scale. The robust electric contact were proved by bias-XPS measurement on a MOS structure. It was also proved that there was no detectable contamination or damage by the contact. A non-destructive robust electric contact with the probe has been proved effective also for $2 \mathrm{D}$ films such as graphene.

\section{ACKNOWLEDGMENTS}

This study is supported by Grants-in-Aid from the Ministry of Education and Science of Japan (No. 25420306).
[1] J. Robertson, Pep. Prog. Phys. 69, 327 (2006).

[2] G. Ribes, J. Mitard, M. Denais, S. Bruyere, F. Monsieur, C. Parthasarathy, E. Vincent, and G. Ghibaudo, IEEE Trans. Device Mater. Reliability, 5, 5 (2005).

[3] M Houssa (ed.), High $k$ Gate Dielectrics (IOP Publishing Ltd, 2004).
[4] Y. C. Yeo, T. J. King, and C. Hu, J. Appl. Phys. 92, 7266 (2002)

[5] J. K. Schaeffer, S. B. Samavedam, D. C. Gilmer, V. Dhandapani, P. J. Tobin, J. Mogab, B.-Y. Nguyen, B. E. White Jr., S. Dakshina-Murthy, R. S. Rai, Z.-X. Jiang, R. Martin, M. V. Raymond, M. Zavala, L. B. La, J. A. Smith, R. 
Garcia, D. Roan, M. Kottke, and R. B. Gregory, J. Vac. Sci. Technol. B 21, 11 (2003).

[6] S. Miyazaki, Appl. Surf. Sci. 190, 66 (2002).

[7] K. Iwamoto, Y. Kamimuta, A. Ogawa, Y. Watanabe, S. Migita, W. Mizubayashi, Y. Morita, M. Takahashi, H. Ota, T. Nabatame, and A. Toriumi, Appl. Phys. Lett., 92, 132907 (2008).

[8] M. Yoshitake, Japanese patent applied. 2006, 296406.

[9] M. Yoshitake, K. Ohmori, and T. Chikyow, Surf. Interface Anal. 42, 70 (2010).

[10] S. Kim, J. Nah, I. Jo, D. Shahrjerdi, L. Colombo, Z. Yao, E. Tutuc and S. K. Banerjee, Appl. Phys. Lett. 94, 062107 (2009).

[11] S. Kim, A. Konar, W.-S. Hwang, J. H. Lee, J. Lee, J. Yang, C. Jung, H. Kim, J.-B. Yoo, J.-Y. Choi, Y. W. Jin, S. Y. Lee, D. Jena, W. Choi, and K. Kim, Nat. Commun.
3, 1011 (2012)

[12] M. Yoshitake, S. Yagyu, and T. Chikyow, Japanese patent applied. 2013, 120634.

[13] Fabricated by Graphene Platform Corp. http:// grapheneplatform.com/

[14] D. C. Giancoli, Physics, 4th Ed (Prentice Hall, 1995).

[15] https://www.nde-ed.org/GeneralResources/ MaterialProperties/ET/ET_matlprop_Misc_Matls.htm (NDT Resource Center).

[16] H. O. Pierson, Handbook of carbon, graphite, diamond, and fullerenes: properties, processing, and applications (William Andrew, 1993).

[17] http://yamanoya.ecs.cst.nihon-u.ac.jp/Thesis/ $\% \mathrm{E} 9 \% 87 \% 8 \mathrm{D} \% \mathrm{E} 6 \% 9 \mathrm{C} \% \mathrm{AC} . \mathrm{pdf}$

[18] http://www.azom.com/news.aspx?newsID=11679 (article published in 2008). 